
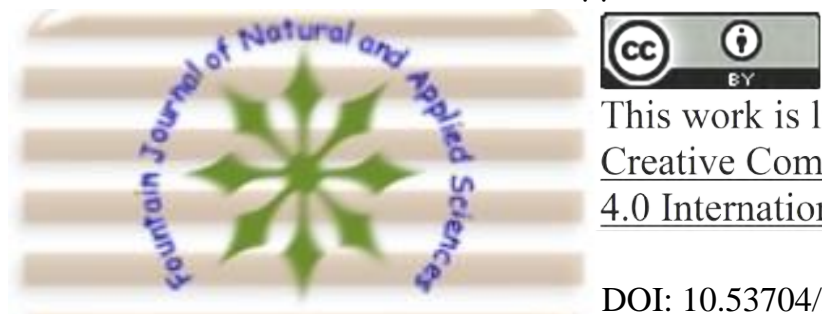

This work is licensed under Creative Commons Attribution 4.0 International License.

DOI: $10.53704 /$ fujnas.v5i2.102

A publication of College of Natural and Applied Sciences, Fountain University, Osogbo, Nigeria. Journal homepage: www. fountainjournals.com

ISSN: 2354-337X (Online), 2350-1863 (Print)

\title{
Orthogonal-Based Second Order Hybrid Initial Value Problem Solver
}

${ }^{1 *}$ Adeyefa, E.O.. ${ }^{1}$ Akinola, L.S., ${ }^{2}$ Folaranmi, R.O. and ${ }^{2}$ Owolabi Y.T.

${ }^{1}$ Department of Mathematics, Federal University Oye-Ekiti, P.M.B. 373, Oye-Ekiti, Ekiti State, Nigeria,

${ }^{2}$ Department of Mathematics, University of Ilorin, P.M.B 1515, Ilorin, Kwara State, Nigeria

\begin{abstract}
This work focuses on development of an initial value problem solver by employing a new class of orthogonal polynomial, the basis function. We present the recursive formula of the class of polynomials constructed and adopt collocation technique to develop the method. The method was analyzed for its basic properties and findings show that the method is accurate and convergent.
\end{abstract}

AMS Subject Classification: 65L05, 65L06

Keywords: Orthogonal polynomials, Algorithm, Block method, Collocation, Interpolation, Zero-Stable.

\section{Introduction}

The second order differential equations arise in many important area of physical problems. The difficulties encounter in solving such problems has led to development of numerical methods. To develop such numerical methods, polynomial plays an important role. Notable among the well-known polynomials are the orthogonal polynomials. Orthogonal polynomial sequence is a family of polynomials such that any two different polynomials in the sequence are orthogonal to each other under some inner product. The first orthogonal polynomials were the Legendre polynomials. Then came the Chebyshev polynomials, the general Jacobi polynomials, the Hermite and the Laguerre polynomials. All these classical orthogonal polynomials play an important role in many applied problems.

Asymptotic formulae for orthogonal polynomials were first discovered bySzegö, (1975). Lanczos (1938) introduced Chebyshev polynomials as trial function. Several researchers have employed these polynomials as trial functions to formulate algorithms (see Shampine and Watts (1969), Tanner (1979), Dahlquist (1979), Jator (2007), Awoyemi (1991)) which are in block forms for solving second order initial value problems directly.

Fatunla (1994) gave a generalization to blockmethods using some definition inmatrix form. Hence, thismotivates us to extend themethod to blockmethod in solving ODEs using a new set of polynomials.

In this work, we shall employ a non-negative weight function to construct a class of orthogonal polynomials which will serve as trial functions to formulate numerical algorithms for the solution of initial value problems.

\section{Construction of Orthogonal Basis Function}

We define the orthogonal polynomial of the first kind of degree $n$ over the interval $[-1,1]$ with respect to weight function $w(x)=\left(x^{2}-1\right)^{2}$ as

*Corresponding author: +2348034150709

Email address: adeoluman@yahoo.com 
Fountain Journal of Natural and Applied Sciences: 2016; 5(2): 12 - 17

$q_{r}(x)=\sum_{r=0}^{n} C_{r}^{(n)} x^{r}$

The following requirements are considered:

$<q_{m}(x), q_{n}(x)>=0, \quad m=0,1,2, \ldots, n-1$

For the purpose of constructing the basis function, we adopt the approach discussed extensively in Adeyefa and Adeniyi (2015) and use additional property (the normalization)

$q_{n}(1)=1$

Using (2), equation (1) yields

$$
\left.\begin{array}{l}
q_{0}(x)=1 \\
q_{1}(x)=x \\
q_{2}(x)=\frac{1}{6}\left(7 x^{2}-1\right) \\
q_{3}(x)=\frac{1}{2}\left(3 x^{3}-x\right) \\
q_{4}(x)=\frac{1}{16}\left(33 x^{4}-18 x^{2}+1\right) \\
q_{5}(x)=\frac{1}{48}\left(143 x^{5}-110 x^{3}+15 x\right) \\
q_{6}(x)=\frac{1}{32}\left(143 x^{6}-143 x^{4}+33 x^{2}-1\right) \\
q_{7}(x)=\frac{1}{32}\left(221 x^{7}-273 x^{5}+91 x^{3}-7 x\right) \\
q_{8}(x)=\frac{1}{384}\left(4199 x^{8}-6188 x^{6}+2730 x^{4}-364 x^{2}+7\right) \\
q_{9}(x)=\frac{1}{128}\left(2261 x^{9}-3876 x^{7}+2142 x^{5}-420 x^{3}+21 x\right) \\
q_{10}(x)=\frac{1}{256}\left(7429 x^{10}-14535 x^{8}+9690 x^{6}-2550 x^{4}+225 x^{2}-3\right)
\end{array}\right\}
$$

In the spirit of Golub and Fischer (1992), equation (3) must satisfy three-term recurrence relation

$$
c_{j} p(t)=\left(t-a_{j}\right) p_{j-1}(t)-b_{j} p_{j-2}(t), j=1,2, \ldots, p_{-1}(t)=0, p_{0}(t) \equiv p_{0}
$$

where

$$
b_{j}, c_{j}>0 \text { for } j \geq 1 \text { ( } b_{1} \text { is arbitrary). }
$$

$c_{j} p(t)=(n+5) P_{n+1}(x),\left(t-a_{j}\right) p_{j-1}(t)=(2 n+5) x P_{n}(x), b_{j} p_{j-2}(t)=n P_{n-1}(x), n=1,2, \ldots$ The recursive formula for these orthogonal polynomials is therefore given as $P_{n+1}(x)=\frac{1}{n+5}\left[(2 n+5) x P_{n}(x)-n P_{n-1}(x)\right], n \geq 1, P_{0}(x)=1, P_{1}(x)=x$

This relation, along with the two polynomials $P_{0}(x)$ and $P_{1}(x)$, allows the new set of polynomials to be generated recursively.

In what immediately follows, we shall develop an algorithm to integrate second order differential equations where polynomials $q_{n}(x)$ shall be employed as basis function. Thereafter, the analysis of the method for convergence and

implementation of the method through some test problems shall be presented. Finally, conclusion shall be made.

\section{Development of the Method}

In this section, our aim is to derive a continuous scheme from which a set of block formula is developed. To make this happen, we shall seek

$$
y(x)=\sum_{r=0}^{s+k-1} a_{r} q_{r}(x)
$$

an

approximant

to obtain the solution of second order initial value problems in ordinary differential equations. Transforming $q_{n}(x)$ to interval $[0,1]$, we have $x=\frac{2 X-2 x_{n}-p h}{p h}$, where $\mathrm{p}$ varies as the method to be developed. In this case, $p=3, s$ and $k$ in (4) are points of interpolation and collocation respectively. The procedure involves interpolating (4) at points $s=0, \frac{1}{3}$ and collocating the second derivative of (4) at points $k=0, \frac{1}{5}, \frac{1}{4}, \frac{1}{3}, \frac{1}{2}, 1,2,3$. The $a_{r}, r=0(1) 9$ from the resulting system of equations are obtained as

$$
a_{r}=\alpha_{0} y_{n}+\alpha_{\frac{1}{3} y_{n+\frac{1}{3}}}+h^{2} \sum_{j=0}^{3} \beta_{j} f_{n+j}+h^{2} \beta_{i} f_{n+i}, r=0(1) 9, i=\frac{1}{5}, \frac{1}{4}, \frac{1}{3}, \frac{1}{2}
$$

Substituting (5) into (4) yields the continuous implicit method

$$
y(x)=\alpha_{0} y_{n}+\alpha_{\frac{1}{3}} y_{n+\frac{1}{3}}+h^{2} \sum_{j=0}^{3} \beta_{j} f_{n+j}+h^{2} \beta_{i} f_{n+i}, i=\frac{1}{5}, \frac{1}{4}, \frac{1}{3}, \frac{1}{2}
$$

Evaluating equation (6) at $x=x_{n+m}, m=\frac{1}{5}, \frac{1}{4}, \frac{1}{2}, 1,2,3$ yields the discrete equations

$$
y_{n+m}=\alpha_{0} y_{n}+\alpha_{\frac{1}{3} y_{n+\frac{1}{3}}}+h^{2} \sum_{j=0}^{3} \beta_{j} f_{n+j}+h^{2} \beta_{i} f_{n+i}, i=\frac{1}{5}, \frac{1}{4}, \frac{1}{3}, \frac{1}{2}
$$

whose values of $\alpha$ and $\beta$ are given in Table 1 .

To develop the block method from the continuous scheme, we adopt the general block formula proposed in Shampine and Watts (1969) in the normalized form given as 
Fountain Journal of Natural and Applied Sciences: 2016; 5(2): 12 - 17

$A^{(0)} Y_{m}=e y_{m}+h^{\mu-\lambda} d f\left(y_{m}\right)+h^{\mu-\lambda} b F\left(y_{m}\right)$

Evaluating the first derivative of (6) at $x=x_{n+j}, j=$ $0, \frac{1}{5}, \frac{1}{4}, \frac{1}{3}, \frac{1}{2}, 1,2,3$, substituting the

$$
\left.\begin{array}{l}
y_{n+k}=\alpha_{0} y_{n}+\psi_{0} h y_{n}^{\prime}+h^{2} \sum_{j=0}^{3} \beta_{j} f_{n+j}+h^{2} \beta_{i} f_{n+i}, k=\frac{1}{5}, \frac{1}{4}, \frac{1}{3}, \frac{1}{2}, 1,2,3, i=\frac{1}{5}, \frac{1}{4}, \frac{1}{3}, \frac{1}{2} \\
y_{n+k}^{\prime}=\psi_{0} y_{n}^{\prime}+h \sum_{j=0}^{3} \beta_{j} f_{n+j}+h \beta_{i} f_{n+i}, k=\frac{1}{5}, \frac{1}{4}, \frac{1}{3}, \frac{1}{2}, 1,2,3, i=\frac{1}{5}, \frac{1}{4}, \frac{1}{3}, \frac{1}{2}
\end{array}\right\}
$$

\section{Analysis of the Method}

\section{Order and Error Constant}

Following Henrici (1962), the approach adopted in Fatunla $(1991,1994)$ and Lambert (1973), we define the local truncation error associated with equation (7) by the difference operator

$$
L[y(x): h]=\sum_{j=0}^{k}\left[\alpha_{j} y\left(x_{n}+j h\right)-h^{2} \beta_{j} f\left(x_{n}+j h\right)\right](10
$$

where $y(x)$ is an arbitrary function, continuously differentiable on $[a, b]$.

Expanding (10) in Taylor series about the point $x$, we obtain the expression

$L[y(x) ; h]=C_{0} y(x)+C_{1} h y^{\prime}(x)+C_{2} h^{2} y^{\prime \prime}(x)+\ldots+C_{p+3} h^{p+3} y^{p+3}(x)$ where the $C_{0}, C_{1}, C_{2}, C_{p}, \ldots$ are obtained as

$C_{0}=\sum_{j=0}^{k} \alpha_{j}, \quad C_{1}=\sum_{j=1}^{k} j \alpha_{j}, \quad C_{2}=\frac{1}{2 !} \sum_{j=1}^{k} j^{2} \alpha_{j}$, $C_{q}=\frac{1}{q !}\left[\sum_{j=1}^{k} j^{q} \alpha_{j}-q(q-1)(q-2) \sum_{j=1}^{k} \beta_{j} j^{q-3}\right]$

According to Lambert (1973), equations (7) is of order $p$ if

$C_{0}=C_{1}=C_{2}=\ldots C_{p}=C_{p+1}=0$ and $C_{p+2} \neq 0$

The $C_{p+2} \neq 0$ is called the error constant and $C_{p+2} h^{p+2} y^{p+2}\left(x_{n}\right)$ is the principal local truncation error at the point $x_{n}$.
Thus, equations (7) is of order 8 with the error constants

$C_{p+2}=\left[7.76 \times 10^{-11}, 4.89 \times 10^{-11},-5.25 \times 10^{-11},-2.59 \times 10^{-8}, 7.22 \times 10^{-6},-1.22 \times 10^{-4}\right]^{T}$

\section{Zero Stability of the Method}

According to Lambert (1973), a linear multistep method is said to be zero-stable if no root of the first characteristic polynomial $\rho(R)$ has modulus greater than one and if every root of modulus one has multiplicity not greater than the order of the differential equation.

To analyze the zero-stability of the method, we present (9) in vector notation form of column vectors $e=\left(e_{1} \ldots e_{r}\right)^{T}, \quad d=\left(d_{1} \ldots d_{r}\right)^{T}$, $y_{m}=\left(y_{n+1} \ldots y_{n+r}\right)^{T}, F\left(y_{m}\right)=\left(f_{n+1} \ldots f_{n+r}\right)^{T}$ and matrices $A=\left(a_{i j}\right), B=\left(b_{i j}\right)$.

Thus, equation (9) forms the block formula $A^{0} y_{m}=h B F\left(y_{m}\right)+A^{1} y_{n}+h d f_{n}(11)$ where $h$ is a fixed mesh size within a block.

Hence, based on the definition above, the scheme is zero stable.

\section{Consistency of the Method}

According to Lambert (1973), a linear multistep method is said to be consistent if it has order at least one. Owing to this definition, equations (7) and (9) are consistent. 
Fountain Journal of Natural and Applied Sciences: 2016; 5(2): 12 - 17

\section{Convergency of the Method}

According to the theorem of Dahlquist, the necessary and sufficient condition for a LMM to be convergent is to be consistent and zero stable. Since the method satisfies the two conditions hence it is convergent.

\subsection{Numerical Experiment}

Three test problems are considered to demonstrate the accuracy of the method.

Problem 1: $y^{\prime \prime}=-1001 y^{\prime}-1000 y, y(0)=1, y^{\prime}(0)=-1, h=0.05$

Exact Solution: $y(x)=\exp (-x)$

Problem 2:

$y^{\prime \prime}=-\frac{6}{x} y^{\prime}-\frac{4}{x^{2}} y, y(1)=1, y^{\prime}(1)=1, h=\frac{0.1}{32}$

Exact Solution: $y(x)=\frac{5}{3 x}-\frac{2}{3 x^{4}}$

Problem 3: We consider Vander pol's Oscillator Problem $y^{\prime \prime}=2 \cos x-\cos ^{3} x-y^{\prime}-y-y^{2} y^{\prime}, y(0)=0, y^{\prime}(0)=1, h=0.1$ whose exact solution is $y(x)=\sin x$

Table 1: Tabular Representation of Discrete Equations

\begin{tabular}{|c|c|c|c|c|c|c|}
\hline \multicolumn{2}{|r|}{$y_{n+\frac{1}{5}}$} & $y_{n+\frac{1}{4}}$ & $y_{n+\frac{1}{2}}$ & $y_{n+1}$ & $y_{n+2}$ & $y_{n+3}$ \\
\hline \multirow{2}{*}{$f_{n}$} & -559066591 & -146852003 & 4222913 & 258407 & 6193885 & 23653037 \\
\hline & 645848437500 & 270888468480 & 4232632320 & 8266860 & 1653372 & 688905 \\
\hline \multirow{2}{*}{$f_{n+\frac{1}{5}}$} & 128106749 & -10505023435 & $152527343 B$ & 210859375 & 11159289085 & 19658828125 \\
\hline & 5208121800 & $682638940 \$ 96$ & 42664933786 & 208324872 & 833299488 & 17360406 \\
\hline \multirow{2}{*}{$f_{n+\frac{1}{4}}$} & $238680160 \Xi 6$ & 208524331 & 4832648 & 328042496 & 688990208 & 104746409984 \\
\hline & 1243258242875 & 20369543040 & 159137055 & 159137055 & 2893401 & 53045685 \\
\hline \multirow{2}{*}{$f_{n+\frac{1}{3}}$} & 91280227 & 5095663 & 1045799 & 36593 & 1580795 & 2625347 \\
\hline & $118125000(0)$ & 990904320 & 30965760 & 30240 & 12096 & 2520 \\
\hline \multirow{2}{*}{$f_{n+\frac{1}{2}}$} & 1617428144 & 2159021 & 299791 & 2781328 & 28511696 & 355815616 \\
\hline & 242193164625 & 5079158784 & 198404640 & 6200145 & 1240029 & 2066715 \\
\hline \multirow{2}{*}{$f_{n+1}$} & 5594563 & -1483733 & 103099 & 119087 & 24778813 & 2953843 \\
\hline & $645848437 \$ 0$ & 27088846840 & 3386105850 & 8266860 & 13226976 & 688905 \\
\hline \multirow[t]{2}{*}{$f_{n+2}$} & 21226229 & 1124087 & -3103 & 28073 & 5553811 & 11209049 \\
\hline & 20344225782500 & 170659735 \#240 & 53331167232 & 520812180 & 104162436 & 8680203 \\
\hline \multirow{2}{*}{$f_{n+3}$} & -6957967 & -368323 & 2203 & 7723 & -123143 & 4323101 \\
\hline & $198921318 \not 00000$ & 16686729683680 & 104292060348 & 5092385760 & 185177664 & 84873096 \\
\hline
\end{tabular}


Fountain Journal of Natural and Applied Sciences: 2016; 5(2): 12 - 17

Table 2: Numerical Results of Problem 1

\begin{tabular}{|l|l|l|l|l|}
\hline \multicolumn{1}{|c|}{$x$} & \multicolumn{1}{|c|}{ Exact } & \multicolumn{1}{c|}{ New Method } & \multicolumn{1}{c|}{ Error } & \multicolumn{1}{c|}{ Error in [2] } \\
\hline 0.05 & 0.95122942450071400909 & 0.95122942450071076950 & $3.23959 e-15$ & $2.05 e-11$ \\
\hline 0.1 & 0.90483741803595957316 & 0.90483741803594163261 & $1.794055 e-14$ & $4.39 e-11$ \\
\hline 0.15 & 0.86070797642505780723 & 0.86070797642498871168 & $6.909555 e-14$ & $6.55 e-11$ \\
\hline 0.2 & 0.81873075307798185867 & 0.81873075307774470455 & $2.3715412 e-13$ & $8.38 e-11$ \\
\hline 0.25 & 0.77880078307140486825 & 0.77880078307062386240 & $7.8100585 e-13$ & $9.86 e-11$ \\
\hline 0.03 & 0.74081822068171786607 & 0.74081822067918416587 & $2.5337002 e-12$ & $1.10 e-10$ \\
\hline 0.35 & 0.70468808971871343435 & 0.70468808971053769036 & $8.17574399 e-12$ & $1.19 e-10$ \\
\hline 0.4 & 0.67032004603563930074 & 0.67032004600930714374 & $2.6332157 e-11$ & $1.24 e-10$ \\
\hline 0.45 & 0.63762815162177329314 & 0.63762815153701783346 & $8.475545968 e-11$ & $1.28 e-10$ \\
\hline 0.5 & 0.60653065971263342360 & 0.60653065943988919902 & $2.7274422458 e-10$ & $1.30 e-10$ \\
\hline
\end{tabular}

Table 3: Numerical Results of Problem 2

\begin{tabular}{|l|l|l|l|l|}
\hline \multicolumn{1}{|c|}{$\mathrm{X}$} & \multicolumn{1}{|c|}{ Exact } & \multicolumn{1}{c|}{ New Method } & \multicolumn{1}{c|}{ Error } & \multicolumn{1}{c|}{ Error in [4] } \\
\hline 0.003125 & 1.00307652585769622630 & 1.00307652585769623090 & $4.6 e-18$ & $3.8354 \mathrm{E}-05$ \\
\hline 0.00625 & 1.00605750308351628300 & 1.00605750308351632460 & $4.16 e-17$ & $7.5004 \mathrm{E}-05$ \\
\hline 0.009375 & 1.00894499508883757910 & 1.00894499508883768810 & $1.09 e-16$ & $1.0592 \mathrm{E}-04$ \\
\hline 0.0125 & 1.01174101816798852880 & 1.01174101816798873410 & $2.053 e-16$ & $1.35476 \mathrm{E}-04$ \\
\hline 0.015625 & 1.01444754268641387440 & 1.01444754268641420320 & $3.288 e-16$ & $1.55567 \mathrm{E}-04$ \\
\hline 0.01875 & 1.01706649423567260840 & 1.01706649423567308660 & $4.782 e-16$ & $1.86372 \mathrm{E}-04$ \\
\hline 0.021875 & 1.01959975475628759200 & 1.01959975475628824410 & $6.521 e-16$ & $1.96055 \mathrm{E}-04$ \\
\hline 0.025 & 1.02204916362943174130 & 1.02204916362943259040 & $8.491 e-16$ & $2.21045 \mathrm{E}-04$ \\
\hline 0.028125 & 1.02441651873840268050 & 1.02441651873840374840 & $1.0679 e-15$ & $2.05628 \mathrm{E}-04$ \\
\hline 0.03125 & 1.02670357750080598400 & 1.02670357750080729130 & $1.3073 e-15$ & $2.77908 \mathrm{E}-04$ \\
\hline
\end{tabular}

Table 4: Numerical Results of Problem 3

\begin{tabular}{|c|l|l|l|}
\hline$X$ & \multicolumn{1}{|c|}{ Exact } & \multicolumn{1}{c|}{ New Method } & \multicolumn{1}{c|}{ Error } \\
\hline 0.1 & 0.09983341664682815231 & 0.09983341664641143268 & $4.16719627 e-13$ \\
\hline 0.2 & 0.19866933079506121546 & 0.19866933079151260797 & $3.54860749 e-12$ \\
\hline 0.3 & 0.29552020666133957511 & 0.29552020665229235391 & $9.0472212 e-12$ \\
\hline 0.4 & 0.38941834230865049167 & 0.38941834229214808125 & $1.650241042 e-11$ \\
\hline 0.5 & 0.47942553860420300027 & 0.47942553857875939095 & $2.544360932 e-11$ \\
\hline 0.6 & 0.56464247339503535720 & 0.56464247335967945648 & $3.535590072 e-11$ \\
\hline 0.7 & 0.64421768723769105367 & 0.64421768719198266396 & $4.570838971 e-11$ \\
\hline 0.8 & 0.71735609089952276163 & 0.71735609084353295021 & $5.598981142 e-11$ \\
\hline 0.9 & 0.78332690962748338846 & 0.78332690956173874562 & $6.574464284 e-11$ \\
\hline 1.0 & 0.84147098480789650665 & 0.84147098473329359276 & $7.460291389 e-11$ \\
\hline
\end{tabular}

\section{Discussion of Results}

Tables 2, 3 and 4 give the numerical results for problems 1,2 and 3 . The superiority of our method is established numerically in Tables 2 and 3 as it compared favourably well with existing methods.

The efficiency of the method, which we further implement on Vander pol's Oscillator
Problem, is seen in Table 4 as it reproduces the exact solutions with small error values.

\section{Conclusion}

Formulation of initial value problem solver has been developed using a new class orthogonal polynomials with recursive formula. Three test problems have been considered to show the 
Fountain Journal of Natural and Applied Sciences: 2016; 5(2): 12 - 17

efficiency and accuracy of the method.

Tables 2, 3 and 4 display the accuracy and comparison of the numerical results of the method with existing methods. The method is desirability as its superiority has been established by the numerical results.

We hope to extend the scope of this study to partial differential equation in our future paper.

\section{References}

Adeyefa, E.O., \& Adeniyi, R.B. (2015) Construction of Orthogonal Basis Function and formulation of continuous hybrid schemes for the solution of third order ODEs. Journal of the Nigerian Association of Mathematical Physics, 29, 21-28.

Adeniran, A.O., \& Ogundare, B.S. (2015) An efficient hybrid numerical scheme for solving general second order initial value problems (IVPs). International Journal of Applied Mathematical Research, 4(2), 411419.

Awoyemi, D.O. (1991) A class of Continuous Linear Multistep Method for general second order initial value problems in ordinary differential equations. International Journal of Computer Mathematics, 72, 2937.

Badmus, A.M., \& Yahaya, Y.A. (2009) An Accurate Uniform Order 6 Block Method for Direct Solution of General Second Order Ordinary Differential Equations. The Pacific Journal of Science and Technology, 10(2), 248-254.

Dahlquist, G. (1979) Some properties of linear multistep and one leg method for ordinary differential equations. Department of computer science, Royal institute of technology, Stockholm.
Fatunla, S.O. (1991) Block Method for Second Order Initial Value Problem (IVP). International Journal of Computer Mathematics, 41, 55-63.

Fatunla, S.O. (1994) A Class of Block Methods for Second Order IVPs. International Journal of Computer Mathematics, 55, 119-133.

Golub, G.H., \& Fischer B. (1992) How to generate unknown polynomials out of known orthogonal polynomials. Journal of Computational and Applied Mathematics, 43, $99-115$.

Jator, S.N. (2007) A sixth order Linear Multistep Method for direct solution of $y^{\prime \prime}=f\left(x, y, y^{\prime}\right)$. International Journal of Pure and Applied Mathematics, 40(1), 407472.

Henrici, P. (1962) Discrete variable methods for ODEs. John Wiley, New York.

Lambert, J.D. (1973) Computational methods for ordinary differential equations. John Wiley, New York.

Lanczos, C. (1938) Trigonometric interpolation of empirical and analytical functions. Journal of Mathematical Physics. 17, 123 - 199.

Shampine, L.F., \& Watts, H.A. (1969) Block implicit one-step methods. Journal of Computer Mathematics. 23:731-740.

Szego, G. (1975) Orthogonal polynomials. American Mathematical Society Colloquium publications.

Tanner, L.H. (1979) The spreading of Silicone Oil Drops on Horizontal Surfaces. Journal of Physics and Applied Physics 12:1473-1484. 